Bundesgesundheitsbl -

Gesundheitsforsch - Gesundheitsschutz

2001·44:944@ Springer-Verlag 2001
Bekanntmachung des Umweltbundesamtes

\section{Einstufung \\ wassergefährdender Stoffe}

ie Kommission Bewertung wassergefährdender Stoffe (KBwS) beim Bundesministerium für Umwelt, Naturschutz und Reaktorsicherheit (BMU) hat auf ihrer 2. Sitzung 2001 für folgende Stoffe Wassergefährdungsklassen (WGK) festgelegt:

\section{Neueinstufungen abweichend von Anhang 3 der VwVwS vom 17.5.1999:}

\begin{tabular}{llll} 
CAS-Nummer & Stoffbezeichnung & Kenn-Nummer & WGK \\
\hline $103-24-2$ & Bis(2-ethylhexyl)azelat & 3772 & nwg \\
$292-64-8$ & Cyclooctan & 3842 & 2 \\
$590-19-2$ & $1,2-B u t a d i e n$ & 3731 & 1
\end{tabular}

\section{Dokumentationen gemäß Anhang 3 der VwVwS vom 17.5.1999:}

\begin{tabular}{llll} 
CAS-Nummer & Stoffbezeichnung & Kenn-Nummer & WGK \\
\hline $1314-13-2$ & Zinkoxid & 2187 & 2
\end{tabular}

Diese Bewertungen werden dem Bundesministerium für Umwelt, Naturschutz und Reaktorsicherheit (BMU) zur Bekanntmachung in der nächsten Fortschreibung der Verwaltungsvorschrift wassergefährdende Stoffe $(\mathrm{VwVwS})$ vorgeschlagen.

Einsprüche und Rückfragen sind zu richten an:

Geschäftsstelle der Kommission Bewertung wassergefährdender Stoffe (KBwS) im Umweltbundesamt, Schichauweg 58, 12307 Berlin,

Postanschrift: Postfach 33 oo 22, 14191 Berlin 